\title{
A georeferenced agent-based model to analyze the climate change impacts on ski tourism at a regional scale
}

\author{
Marc Pons $^{\mathrm{a}, \mathrm{b}^{*}}$, Peter A Johnson ${ }^{\mathrm{c}}$, Martí Rosas-Casals ${ }^{\mathrm{b}}$ and Eric Jover ${ }^{\mathrm{a}}$ \\ ${ }^{a}$ Observatori de la Sostenibilitat d'Andorra, Sant Julià de Lòria, Andorra; ${ }^{b}$ Sustainability \\ Measuring and Modelling Lab, Universitat Politècnica de Catalunya, BarcelonaTech, \\ Terrassa,Barcelona, Spain; \\ ${ }^{c}$ Department of Geography and Environmental Management, University of Waterloo, \\ Waterloo, Ontario, Canada
}

(Received 21 January 2014; final version received 1 June 2014)

\section{ABSTRACT}

One main argument for modeling socio-ecological systems is to advance the understanding of dynamic correlations among various human and environmental factors, including impacts and responses to environmental change. We explore the shift in skier distribution amongst ski resorts taking into account the behavioral adaptation of individuals due to the impact of climate change on snow conditions. This analysis is performed at a regional scale by means of a coupled gravity and georeferenced agentbased model. Four different scenarios are considered. Two scenarios assume an increase of winter mean temperature of $+2^{\circ} \mathrm{C}$ and $+4^{\circ} \mathrm{C}$ respectively, taking into account only natural snow conditions. Two additional scenarios add the effect of snowmaking to enhance the natural snow depth and extend the skiing season in the $+2^{\circ} \mathrm{C}$ and $+4^{\circ} \mathrm{C}$ base scenarios. Results show differing vulnerability levels, allowing the classification of ski resorts into three distinct groups: (1) highly vulnerable ski resorts with a strong reduction in visitors attendance for all climate change scenarios, characterized by unfavorable geographical and attractiveness conditions, making it difficult to ensure snow availability in the future; (2) low vulnerability ski resorts, with moderate reduction in season length during a high climate change scenario but no reduction (or even an increase) in a low one, characterized by ski resorts with a medium capacity and attractiveness to ensure enough snow conditions and capture skiers from other ski resorts; and (3) resilient ski resorts, with good conditions to ensure future snow-reliable seasons and outstanding attractiveness, allowing them to offer longer ski seasons than their competitors and potentially attracting skiers from other closed or marginal resorts. Ski resorts included in this last group increase their skier attendance in all climate change scenarios. Although similar studies in the literature foretell a significant reduction of the ski market in the near future, another probable effect outlined in this study is a redefinition of this market due to a redistribution of skiers, from vulnerable ski resorts to more resilient ones.

Key words: Climate Change; Winter Tourism; Adaptation; GIS; Agent Based Model. *Corresponding author. Email: mpons@obsa.ad 


\section{INTRODUCTION}

One main argument for modeling socio-ecological systems is to advance the understanding of the dynamic correlations amongst various human and environmental factors, including impacts and responses to environmental changes. Examples come from a broad spectrum of spatial phenomena such as dynamics in ancient human and primate societies (Kohler et al., 1999, Axtell et al., 2002; Janssen, 2009), land use and land cover change (Manson and Evans, 2007; Parker et al., 2003), water management (Smajgl et al., 2009; Viaggi et al., 2009; Bithell and Brasington, 2009), residential segregation in urban contexts (Crooks, 2010) or insect outbreak spreading (Perez and Dragizevic, 2010). However, few studies have analyzed individual human and environmental interactions in tourism phenomena. Gimblett \& Skov-Petersen (2008) and Itami et al. (2002) in particular, used ABM for the simulation and visualization of movement patterns of visitors in recreational landscapes, such as parks and protected areas. Johnson \& Sieber (2009, 2010 and 2011) developed an ABM of tourism dynamics including travel, lodging and activity patterns. Regarding winter tourism, responses to environmental impacts and changes in tourism dynamics, Pons-Pons et al. (2012) developed a georeferenced ABM to analyze the climate change impacts on the ski winter tourism in Andorra and Balbi et al. (2013) used a spatial agent-based model for assessing strategies of adaptation to climate and tourism demand changes in an alpine tourism destination. In recent years ABM models have been identified as a promising methodology to analyze tourism dynamics (Baggio, 2008). First, because they model and characterize interacting human-nature processes of heterogeneous individual behaviors that occur over space and time (Axtell, et al. 2002, 1996; Parker et al. 2003). In an ABM, tourist agents can be characterized with more realistic heterogeneous behaviors, governing activity, decision or accommodation preferences. For example, the visitor response if a ski resort were closed, as well as spatial characteristics, such as travel distances. Second, this approach is well suited for scenario development, data analysis, problem diagnosis and policy comparison (LigmannZielinska \& Jankowski, 2007; Johnson \& Sieber, 2011). Since ABM facilitates the representation of individual-level spatio-temporal interactions, they have a relevance to representing and understanding the dynamics and characteristics of tourism.

Many studies dealing with tourism are focused on how climate change will affect the supply side of tourism, such as resorts, facilities or season days (Scott et al., 2003, 2008; Becken, 2005; Hoffmann et al., 2009; Steiger, 2010; Pütz et al. 2011). Many models analyzing climate change impacts on ski tourism base their estimations on physical snow models such as Abegg et al., (2007), Scott (2003), Uhlman (2009) or Steiger (2010). In these approaches, variables such as snow depth and duration of simulated snowpack are used to estimate the impact of climate change on the number of operational days (length of season). Some of these studies only model the natural snowpack at ski resorts (Uhlmann et al., 2009) or apply indicators such as snow cover days, defined as $2.5 \mathrm{~cm}$ of snow (Lamothe \& Périard, 1988). This level of cover does 
not match with the reality of ski area operations that require $30-100 \mathrm{~cm}$ of snow to open a ski run. A few studies create statistical relationships between the length of season and snow depth or other climatological parameters (Moen and Fredmand, 2007). One of the major limitations of these studies using statistical models is the omission of the effect of snowmaking on future natural snowpack. This is the main limitation found in most of the previous literature analyzing the vulnerability of ski resorts (Scott et al., 2012). Studies that do incorporate snowmaking (Scott et al., 2003, 2008,; Hennessy et al., 2008; Steiger, 2010) found that impacts on ski resorts are lower than those reported by previous studies that only take into account natural snow. An alternate approach to these statistical and physical models is the analogue approach, where past and present experiences and responses to climatic variability, change and extremes provide insight for vulnerability to future climate change (Ford et al., 2010). This approach has been applied in North America (Scott, 2006; Dawson et al., 2009) and the Austrian region of Tyrol (Steiger, 2011), comparing the impact of climate change scenarios on season length. Most of these previous studies are focused on modeling supply-side impacts (ski operations) and none have explored how ski tourist demand has shifted in response to climate change impacts. Defining the mechanisms and variables related to tourists adaptation and behavior is an immediate research need to be filled. Even though recent studies point out that behavioral adaptation of tourists due to spatial, temporal and activity substitution could exert a strong influence on the final output of the climate change impact assessments (Behringer et al., 2000; Dawson et al., 2011, Gössling et al, 2012; Dawson et al., 2013), there is no analysis of this issue in the literature. This study proposes a new approach to better understand and explore how the potential behavioral responses identified in previous studies could affect the final outcome of climate change impact assessment. In addition to the identified behaviors and responses of tourists to climate change, this framework could be also applied to explore the influence of tourist behavior and responses to other factors that could affect their dynamics such as energy and transport issues or the influence of crowding in destinations, once this information has been gathered.

We explore the shift in tourist distribution amongst Pyrenean ski resorts taking into account the behavioral adaptation of individuals due to the impact of climate change on snow conditions. The Pyrenees is a mountain range comprising the northern portion of Spain, the southern portion of France and the small country of Andorra. Enclosing 49 alpine ski resorts, this region receives around $11 \mathrm{M}$ skiers per year. Most of these skiers come from the surrounding regions. In Andorra, the most international destination in the Pyrenees, almost a $70 \%$ of the skiers come from the surrounding regions of Spain and France. This share is much higher in other ski resorts and turns the Pyrenees into a quite compact market with no significant competitors at close distances. In this context, the Pyrenees are highly dependent on winter tourism industry being their residents well aware that climate change is presented as a future threaten to snow availability and to future development of winter tourism related activities (March et al., 2014). 
This analysis is performed at a regional scale by means of a coupled gravity and agentbased model (ABM). Unlike many models used to date, this methodology includes behavioral responses and heterogeneity of winter tourists. The use of a geo-referenced landscape makes it possible to capture the intrinsic spatial features of tourism phenomena, such as ski resort location and travel distances between them. In addition, the ABM model is linked to a gravity model: the potential redistribution of skiers among ski resorts when some of these have to close due to poor snow conditions, depends on the attractiveness of each ski resort and the travel distance between them.

The paper is organized as follows: in section 2, we present our model description following the "Overview, Design concepts and Details" (ODD) protocol (Grimm et al., 2006; 2010). Section 3 presents the model validation process Section 4 presents the specific scenarios generated in order to assess the vulnerability of the ski industry and the resulting outcome for each of these different projections Section 5 present and discusses the main findings of the paper, the suitability of the methodology, its limitations and the implications for further work. Finally section 6 presents the conclusions of this study.

\section{MATERIALS AND METHODS}

The "Overview, Design concepts and Details" (ODD) protocol, an accepted standard in ecological and social science literature, is used to formulate and describe the agent based model (Grimm et al., 2006; 2010). The overview component if this protocol

provides an explanation about how the model is designed. Purpose, entities and attributes are then described to define the ABM. An entity is a distinct or separate object or actor that behaves as a unit in the ABM and may interact with other entities or be affected by the environment. The current state of the object is characterized by attributes. An attribute is a variable that distinguishes an entity from other entities of the same type or category, or traces how the entity changes over time. The design concepts depict the essential characteristics of the model. Finally the details section describes other information such as input data and submodels needed to understand, reimplement and replicate the model. 


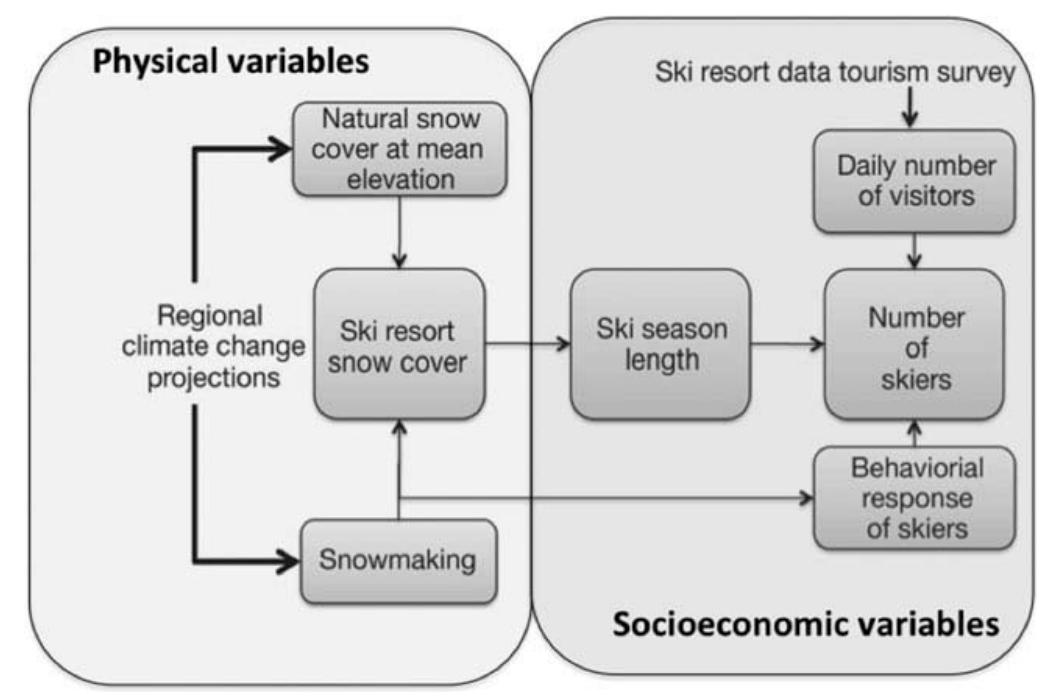

Figure 1. Conceptual map of the model linking the physical and the social variables.

Figure 1 shows the conceptual map of the model. Climate projections of future snow depth and potential snowmaking capacity at the mean elevation of the resort as well as the daily attendance of skiers at each ski resort are used as input data for the model. Using this information the model simulates by means of a gravity model and an ABM, the future ski season length and the daily attendance of skiers at each ski resort at a regional scale. As case study, the ski industry in the Pyrenean region, including France, Spain and Andorra is analyzed.

\subsection{Overview}

\subsubsection{Purpose}

The model is designed to explore questions about the shift in the skier distribution among ski resorts at a regional scale due to future climate change projections affecting local snow conditions at each individual ski resort.

\subsubsection{Entities, state variables, scales and environment}

In this model there are 2 main entities: skiers, the agents of our model, and ski resorts, which are fixed on the landscape. Skiers include the following attributes:

1) Assigned ski resort.

2) Current location: coordinates at each time step. This internal information helps the software to locate the agent at a ski resort on the map at each time step.

3) Adaptation strategy: whether skiers perform spatial or activity substitution when the ski resort is closed. 
Ski resorts include the following attributes:

1) Location coordinates.

2) Season length in days.

3) State: whether the ski resort is open or closed.

4) Maximum capacity of daily skiers.

5) Attractiveness (described in more detail in the submodels section).

The simulation lasts for 151 time steps, the length of a standard winter season (from December $1^{\text {st }}$ to April $30^{\text {th }}$ ), being the length of one time step equivalent to one day.

The environment, i.e. the space where the agents interact and respond to its changes, is implemented using two GIS layers: (1) the 41 main ski resorts of the Pyrenees (representing around the $92 \%$ of the total skiers (DSF, 2012; Biotti, 2013; ATUDEM, 2013; SkiAndorra) and (2) the main roads connecting the ski resorts (Figure2). The dimension of the points in this layer changes over time based on the daily attendance of skiers (the greater attendance the larger the point is shown) and the status attribute of each ski resort, that is, whether it is open (green) or closed (red) as a consequence of the daily projected snowpack conditions. The first 2 layers remain static during the simulation.

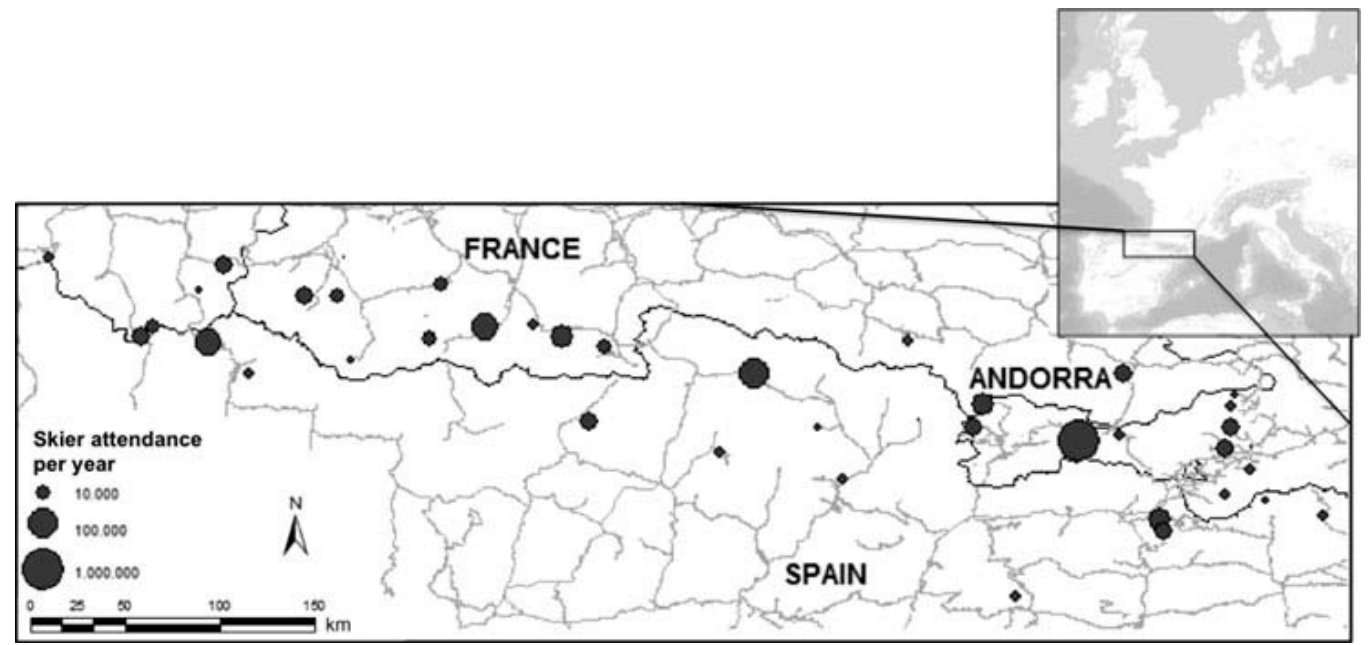

Figure 2. GIS layers with the location of the roads and the ski resorts of the Pyrenees. The size of the bullets represents the average skier attendance at each ski resort.

\subsubsection{Process overview and scheduling}

Using the snow cover projections and potential snowmaking days based on future climate scenarios as input data, the model starts simulating the projected season length at each ski resort and updating daily if it is open or closed during an entire winter season (Figure 3). The aim of this framework is to allow snowpack and ski days simulations to be used and integrated into the ABM regardless of modeling approach used. In our particular case for the Pyrenees, these variables have been simulated using the projected changes in the Pyrenean daily snowpack during the 21 st Century by means of a Snow Energy Balance Model (SEBM) for the study area and coupled with technical 
parameters of ski resorts operation and snowmaking processes. Following the experience of technical staff in ski resorts, a daily minimum temperature threshold of $2{ }^{\circ} \mathrm{C}$ has been used to compute the potential snowmaking days during a winter season. During these potential snowmaking days, it is assumed that a maximum of $10 \mathrm{~cm}$ per day can be produced to reach the $30 \mathrm{~cm}$ threshold. Thus, the natural snow depth is complemented with snowmaking following these criteria. Therefore, a ski resort is considered open as soon as it reaches the $30 \mathrm{~cm}$ threshold considering both natural snow and snowmaking. This threshold is one of the most used criterions to assess the climate change vulnerability of ski resorts (Abegg, 1996; Scott et al. 2008; Steiger et al. 2010). In order to simulate the ski season length, those days with at least $30 \mathrm{~cm}$ of snow depth were those considered as open days. For a detailed description of the snow model used and the climate change projections see López-Moreno et al. (2009) and Pons-Pons et al. (2012). Snowpack projections are based on 2 future emissions scenarios: the IPCC SRES A2 and B2 scenarios for different altitudinal levels: 1500, 2000, 2500 and 3000 $\mathrm{m}$. These scenarios project future climate change based on different assumptions for greenhouse gas emissions, land-use, economic and technological development and other diving forces (IPCC, 2007). In our study B2 and A2 were used as for mid and high climate change scenarios respectively.

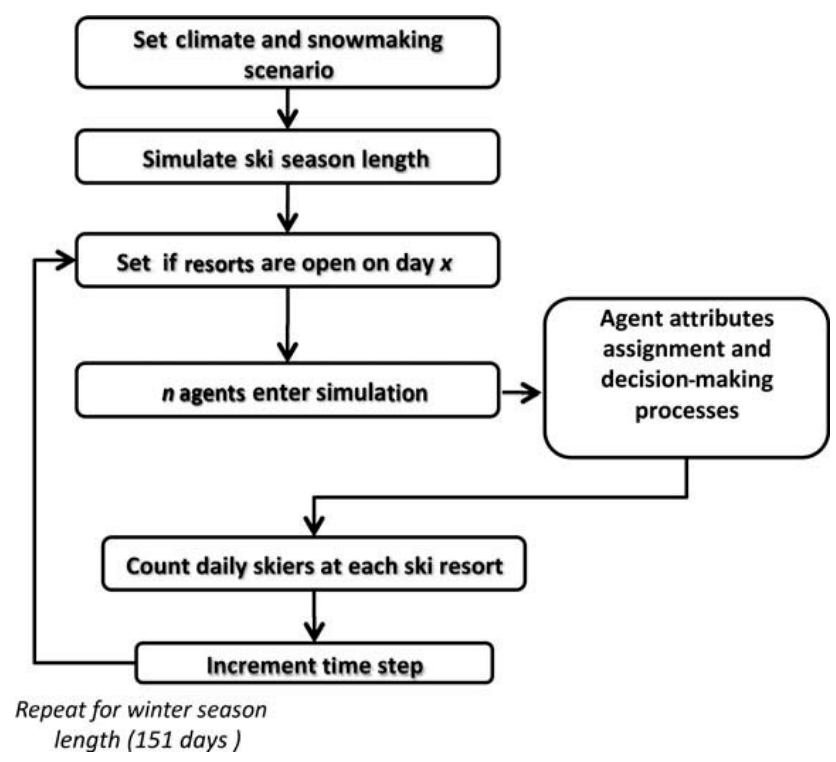

Figure 3. Model processes flowchart.

Based on ski resort statistics and survey data of tourism activity, the model assigns the initial number of agents at each ski resort on the basis of the current distribution of skiers over the studied region. With only aggregated information about skier visits available from official regional ski associations or administrations Statistics (DSF, 2013, ATUDEM, 2013, Botti, 2013), and also considering that many ski resorts do not easily or freely share skier numbers, deep research from more than 20 different local and regional newspapers, ski resorts periodical press releases and news websites was conducted to estimate the frequentation at 41 of the 49 ski resorts of the Pyrenees from 2008-2009 to 2011-2012. The remaining 8 ski resorts are very small ski areas (less than 
a couple or three of runs and a couple of ski lift, often presented as s complementary activity of a hotel or nordic ski resort) which information was not available. From this data, the average from the last 3 seasons was assigned as the average yearly skier visitation at each ski resort. To estimate daily attendance, a daily visitation curve of skiers was estimated in order to modulate the visitation based on holidays, weekends and working days. This issue is a crucial point to the model because the impact of reducing the ski season length is not the same if important holidays such as Easter or Christmas are affected (Steiger, 2013). The only information on tourist visits on a daily basis was provided by the national tourism survey from the Andorran Government and statistics from the national tourism department (Andorra Turisme, 2012) for the seasons 2008-2009 to 2011-2012. These surveys were used to develop the profile of the visitors and identify the daily number of skiers. Approximately 8000 visitors to Andorra respond to the national tourism survey every winter season and this information is linked to the observations of the total foreign vehicles entering the country. Table 1 shows the data and the sources used as input in the model.

\begin{tabular}{|l|l|}
\hline \hline \multicolumn{1}{|c|}{ Variable } & \multicolumn{1}{c|}{ Source } \\
\hline \hline Snow depth projections & Lopez-Moreno et al. 2009 \\
\hline Coordinates of the ski resorts & $\begin{array}{l}\text { GIS layer created by satellite imagery (ICC; } \\
\text { SIGMA; Geoportail France; GoogleEarth) }\end{array}$ \\
\hline $\begin{array}{l}\text { Ski resorts yearly attendance from } \\
\text { 2008-2009 to 2011-2012 seasons }\end{array}$ & $\begin{array}{l}\text { Ski Andorra; Atudem, DSF; Press reports and } \\
\text { newspapers }\end{array}$ \\
\hline Total length of ski slopes & Ski resorts official webpage \\
\hline Price of the day ski pass & Ski resorts official webpage \\
\hline Complementary activities & Ski resorts official webpage \\
\hline Vicinity to commercial area & $\begin{array}{l}\text { GIS layer of cities and villages from France } \\
\text { Andorra and Spain (ICC; SIGMA; Geoportail } \\
\text { France; GoogleEarth) }\end{array}$ \\
\hline Daily attendance curve of skiers & Andorra Turisme; Estadistica.ad \\
\hline
\end{tabular}

Table 1. Data and sources used in the model.

To explore the emergent macro-level phenomena from micro-level individual actions and local conditions of ski resorts, the model implements the adaptation behavior of skiers through spatial and activity substitution (defined in section 2.1.4). Different studies based on surveys (Behringer et al., 2000; Fukushima et al., 2002; Hamilton et al., 2007; Unbehaun et al., 2008; Dawson et al., 2011 and Pütz et al., 2011, Dawson et al, 2013) have identified the potential behavioral and adaptative response of skiers to poor snow conditions. On the basis of these results, the share of potential skiers performing a spatial and an activity substitution are identified and implemented 
in the model. Thus when a ski resort is closed, the decision-making process shown in Figure 4 is applied to each skier at a particular ski resort. These skiers perform an activity substitution and stop skiing when the ski resort where they would typically go skiing is closed. The rest of skiers perform a spatial substitution and are redistributed amongst the remaining open ski resorts in the region by means of a gravity model (described in depth in the submodels section) according to the attractiveness factor of each ski resort and the distance between the origin (in this study, the initially assigned ski resort) and the potential alternative resort.

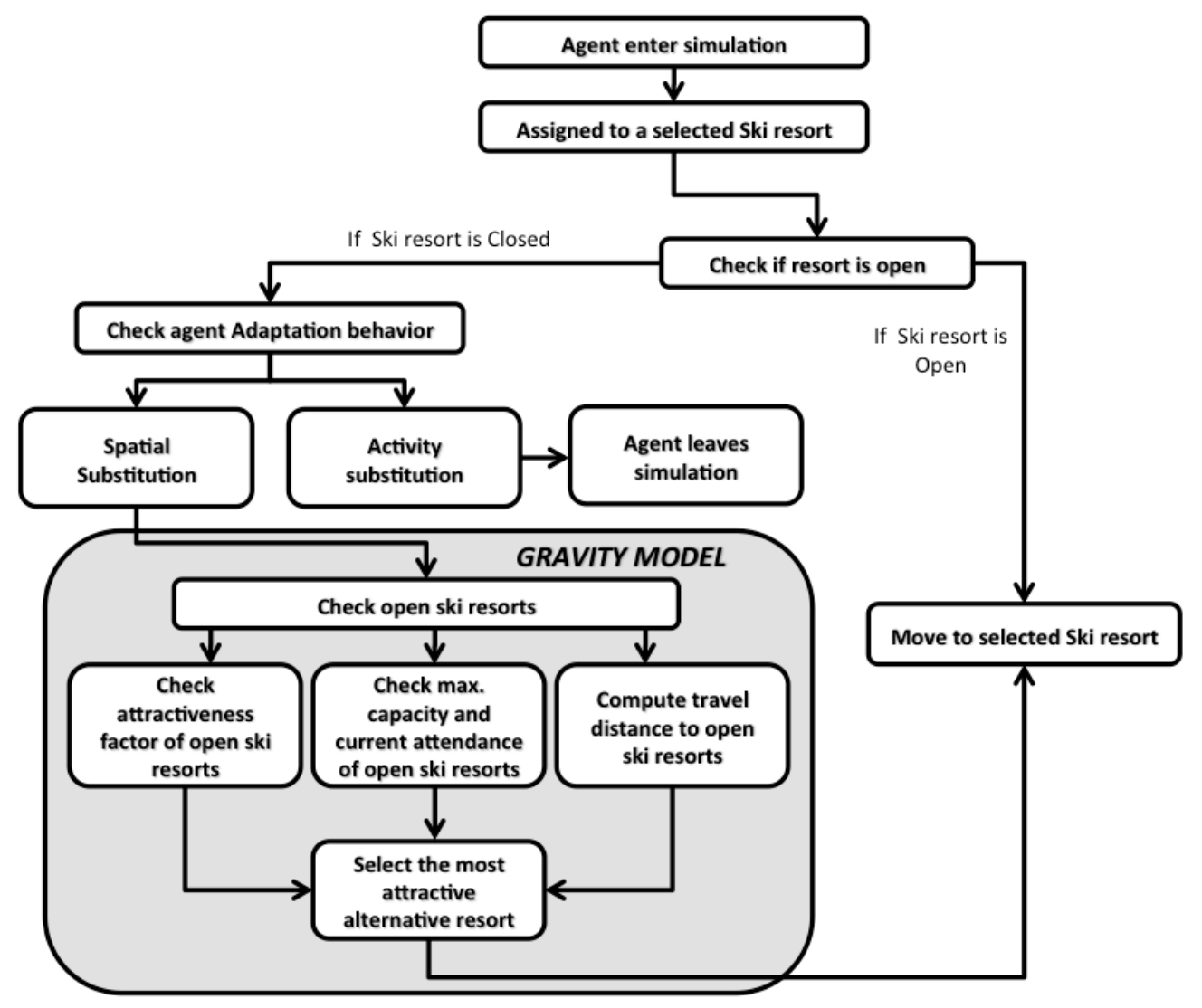

Figure 4. Agent decision-making flowchart.

\subsubsection{Design Concepts}

The basic principle addressed by this model is the emergence of potential shifts in the current skiers distribution at a regional scale due to changes in local snow conditions. This concept is addressed by checking how behavioral adaptation of skiers affects the attendance at the local ski resort level, and at the regional scale, by means of the simulation of the potential redistribution of skiers. Skier agents do not implement any learning or prediction capacity and base their decisions solely on the objective of finding a ski resort with suitable snow conditions to support skiing. To achieve this objective, agents are sensitive to four variables: (1) current snow conditions, (2) travel distance between ski resorts, (3) an attractiveness factor of each ski resort and (4) the 
maximum daily capacity to host skiers. A stochastic process is used to randomly assign the choice of the adaptive behavior (i.e., whether the skiers perform an activity or a spatial substitution). Based on current snow conditions (i.e., the resort is closed due to insufficient snow conditions) agents have a 5\% probability to stop skiing. This probability is based on existing surveys (i.e. Behringer et al, 2000). Otherwise, skiers move to an alternate resort characterized by the gravity model (explained below). Movement to this alternate resort is restricted by its maximum capacity. From interviews with ski resort managers, this maximum capacity was assumed as twice the maximum daily attendance during the reference season (average from 2008/2009 to 2011-2012). This involves an indirect interaction between agents, affecting the selection of the final destination. In this initial version of the model, no collectives have been implemented. However in further versions collectives could be implemented in order to segregate the adaptation behavior and the preference choices of different profile of skiers such as expert skiers, beginners or families. These differences in preference and adaptation behaviors could also affect the potential redistribution of skiers among ski resorts.

To observe shifts in the distribution of skiers at a regional scale, three different variables are analyzed as model output on a daily basis and for each ski resort: (1) number of attracted skiers when open, (2) number of skiers lost when closed due to insufficient snow depth and (3) total seasonal skier attendance.

\subsubsection{Initialization}

The model is initialized for each future scenario with the projected season length of 151 days, with attendance based on the present regional distribution of skiers. The present average distribution of skiers has been calculated as the average of the historical data of attendance for the winter seasons from 2008-2009 to 2011-2012.

\subsubsection{Input data}

The input data of the model are (1) the current daily distribution of skiers at each ski resort and (2) the days with sufficient snow depth (30 $\mathrm{cm}$ indicator), in order to compute the season length during a winter season in different climate scenarios.

\subsubsection{Submodels}

A gravity model, in analogy with Newton's gravity law, is used to analyze the potential redistribution of skiers (spatial substitution) among the ski resorts of the whole region based on both the attractiveness of each ski resort and the travel distance between them when some ski resorts have to close due to poor snow conditions.

Introduced in its contemporary form in 1946 (Zipf, 1946), but with roots that go back to the eighteenth century (Monge, 1781), the gravity law is a spread framework to predict 
population movement (Monge, 1781; Jung et al. 2008; Thiemann et al. 2010) cargo shipping volume (Isard, 1960; Kaluza et al., 2010), inter-city phone calls (Krings et al., 2009), bilateral trade flows between nations (Pöyhönen, 1963) or migration processes (Kararema et al, 2000), just to name a few. The basic principle governing these models is that the shorter the distance between two objects and bigger their mass, the greater the gravitational pull between these two objects. Following this principle, the gravity law assumes that the number of individuals that move between locations $i$ and $j$ is proportional to the mass, i.e. the population of the source and inversely proportional to the distance to the potential destinations.

The model we describe estimates the total number of skiers moving from a closed ski resort $i$ to an open ski resort $j\left(F_{i j}\right)$ on the basis of an attractiveness factor (i.e., mass) of the potential destination resort $\left(G_{j}\right)$ and the inverse of the distance between the origin $i$ and destination resort $\left(D_{i j}\right)$, affected by a unique parameter $\langle$ (Eq. 1).

$$
F_{i j}=G_{j} / D_{i j}^{\alpha} \quad \text { Eq.1 }
$$

The attractiveness factor represents the capacity of each ski resort to attract skiers to their facilities. The create this submodel characterizing this feature, a non-parametric statistical analysis was carried out using a set of 15 physical and socio-economical variables that characterize the ski resorts. Amongst all variables, four were found to be the most significant in explaining the current redistribution of skiers ( $p$-value $<0.05$ ). A multiple regression model was used to identify the main variables that affect the current capacity to attract skiers and explain the present distribution in the region (Eq. 2). The model used, permits to explain almost $90 \%$ of the current distribution of skiers on the Pyrenean region $\left(\mathrm{R}^{2}=0,87\right.$; $\mathrm{p}$-value $\left.<0,001\right)$ based on four variables: (1) total length of ski slopes, (2) the mean cost of the all season daily adult ski pass, (3) whether or not the resort offers complementary activities to skiing, and (4) its location, near or distant from a large commercial area. This last qualitative binary variable assigns a value of 1 to those resorts with a commercial area (not only isolated stores) within a radius of $25 \mathrm{~km}$. This indicator permits to identify those resorts with the ability to offer shopping as a complementary activity, a factor identified to have a high influence on tourists when choosing a ski resort (Andorra Turisme, 2012). All these factors are congruent with previous work that identified, through survey research, the most influential factors when choosing a ski destination (Dawson, 2009).

$$
\begin{gathered}
\text { Attraciveness Factor }=\beta_{1} * \text { Total Lenght km of slopes }+\beta_{2} * \text { mean daily ski pass } \\
+\beta_{3} * \text { Complementary activities }+\beta_{4} * \text { near a commercial center }
\end{gathered}
$$

Eq. 2

By applying the gravity model when a given resort it has to close because of poor snow conditions, we calculate the potential number of skiers that will shift from the closed ski resort to the remaining open resorts. For instance, as Figure 5 shows, if the resort of La Molina is closed (in black), the biggest share of its skiers (46\%) will shift to the nearest 
ski resort, Masella, due to the effect of the distance variable. However, due to the different attractiveness of the resorts, a share of the skiers will move to a further resort. Despite being much further away from La Molina, Grandvalira receives a significant share $(11 \%)$, because of its high attractiveness factor.

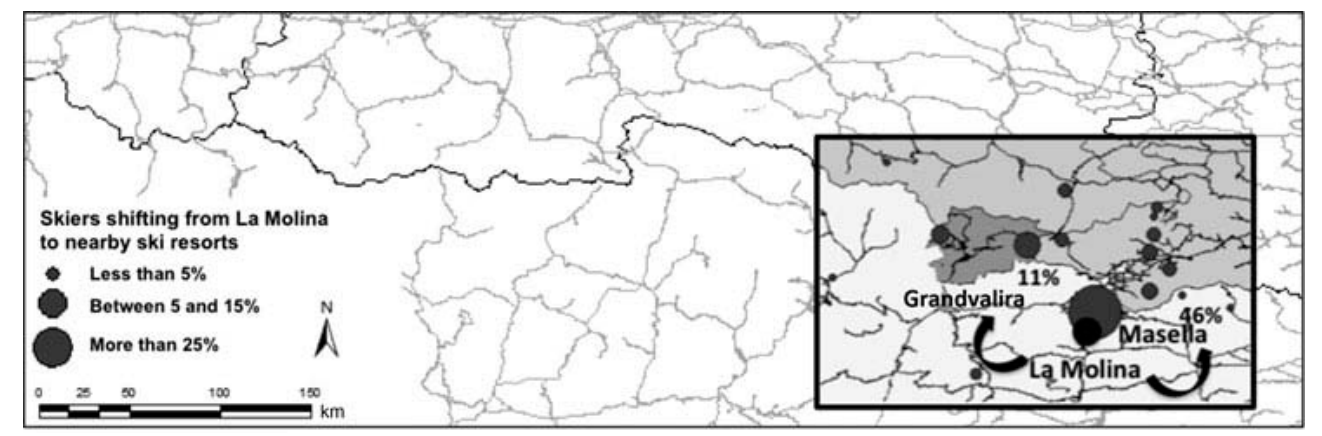

Figure 5. Gravity model applied to the skiers of La Molina when it is closed. The size of the dots represents the percentage of skiers shifting from La Molina (black dot) to nearby resorts.

\section{VALIDATION}

One challenge of this modeling approach is the validation process of the model results. Here we use an analogue approach (Dawson et al., 2009) to corroborate our results, since the outcome of the model are simulations of future skiers attendance based on projected ski season reductions. This approach looks for a past winter season with analogous climate conditions that could reproduce a similar situation as the projected future winter season. However, the low frequency in the occurrence of years with analogous conditions to future climate scenarios and the scarcity of detailed historical data on skiers attendance makes it difficult to carry out a good validation process. In this sense, the 2011-2012 season was found to be the one in the last ten years performing the closest climate situation to the $+2{ }^{\circ} \mathrm{C}$ scenario. During this season, the average minimum temperature in Andorra was $2.2^{\circ} \mathrm{C}$ over the value for the control period (1961-1990), most similar to the $+2{ }^{\circ} \mathrm{C}$ scenario. Thus, the $+2^{\circ} \mathrm{C}$ with snowmaking scenario results are compared to the 2011-2012 skiers attendance, as a form of validation.

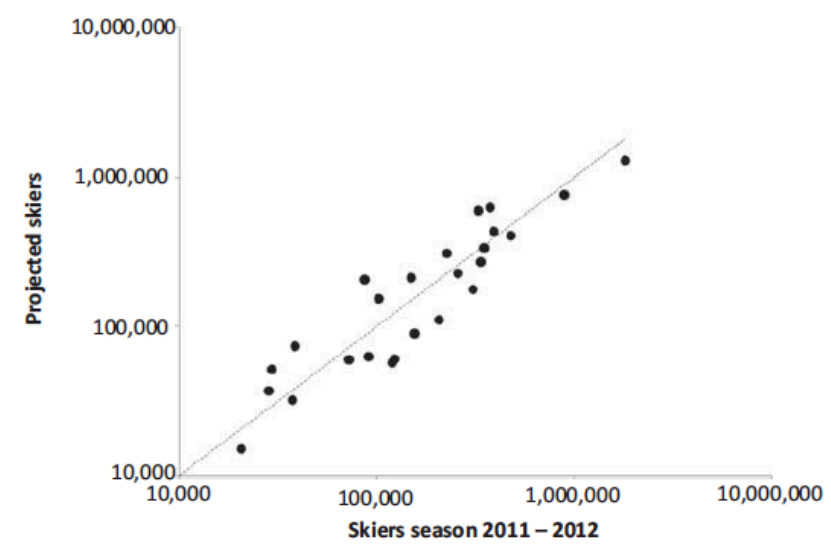

Figure 6. Correlation between real skiers for the season 2011-2012 and projected skiers in the $+2^{\circ} \mathrm{C}$ scenario. 
Figure 6 shows correlated values between projected skiers in the $+2{ }^{\circ} \mathrm{C}$ The model explains remarkably well the real data behavior and no deviation trends are observed. In the resorts where the anomaly of temperature in the analogous year is closer to the projected scenario (central-eastern part of the Pyrenees) the observed error is less than $30 \%$. The error can be explained partly by three main sources:

1) Regional climatic models: there exists a lack of spatial resolution and an inherent uncertainty associated with regional climate models (Alexandru et al., 2007).

2) Quality and quantity of historical data on skiers attendance are not always complete or the desired spatial scale.

3) The model cannot explain temporal substitution (i.e., those skiers not performing spatial substitution, but shifting their skiing season depending on snow availability).

This validation process could be used to assess the usefulness of the gravity approach (eq. 1). This method relies on adjustable parameters to fit empirical data that vary from region to region (Isard, 1960). In this sense, distance $D_{i j}$ is one of the main variables that can be tuned by means of an exponent $\alpha$ (i.e. $D_{i j}{ }^{a}$ ). The best fit result is obtained with $\alpha$ $=0\left(\mathrm{R}^{2}=0.999\right)$, which implies that, in this particular case study, the distance is not a factor having a huge impact on the total distribution of skiers in a future climate change scenario (Figure above). This is mainly due to the fact that in a future climate change scenario only few resorts remain available (the ones with already the highest frequentation). Redistribution options for skiers are reduced and so is sensitivity to alpha in $D_{i j}{ }^{\alpha}$. Thus, the effect of distance is hidden because the amount of skiers shifting to these resilient resorts is low regarding the current high frequentation of these ones. Even though distance effect has not a significant impact on the total distribution, it actually has an impact on the reallocation of skiers (skiers moving because ski closures) shifting from one ski resort to another one. Furthermore, a limitation in this study is that the distance considered is not the distance from the skier home origin to the potential destination but rather the distance between potential destinations. When the model is run under less severe conditions (i.e., both more homogenous attractiveness and heterogeneous variability in the projected season length reduction), $\alpha$ becomes a significant parameter (results not shown in the article). Therefore, $\alpha$ it should be considered and properly tuned in order to apply this model to other regions.

\section{RESULTS}

Four different scenarios were run to analyze the redistribution of future skiers among the Pyrenean ski resorts induced by climate change-related snow reductions. The two 
first scenarios assume an increase of the winter mean temperature of $+2^{\circ} \mathrm{C}$ and $+4^{\circ} \mathrm{C}$ respectively, taking into account only natural snow conditions. The other two scenarios add the effect of the potential snowmaking on enhancing the natural snow depth and extending the ski season length in the $+2^{\circ} \mathrm{C}$ and $+4^{\circ} \mathrm{C}$ base scenarios.

Figure 7 shows the attractiveness factor and the projected number of skiers in a present mean winter season and for two future scenarios: assuming an increase of $+2^{\circ} \mathrm{C}$ and $+4^{\circ} \mathrm{C}$ of the winter mean temperature and taking into account only the natural snow depth to compute the ski season length. Two different groups of ski resorts with different attendance patterns can be identified. For most of the ski resorts $(\approx 70 \%)$, a slight decrease in skier attendance, less than $25 \%$, is projected for a $+2^{\circ} \mathrm{C}$ scenario and a significant decrease, between $50 \%$ and $100 \%$, for the $+4{ }^{\circ} \mathrm{C}$ scenario. However, only few resorts are able to increase the number of visitors in both future scenarios due to both a lower climatic vulnerability and a higher touristic attractiveness compared to their competitors. On the other hand, the current relationship between the attractiveness factor of the ski resorts and the total attendance of skiers per year is almost linear (black line in Figure 7). In a climate change-induced future, this relationship becomes increasingly non-linear (dashed line in Figure 7).

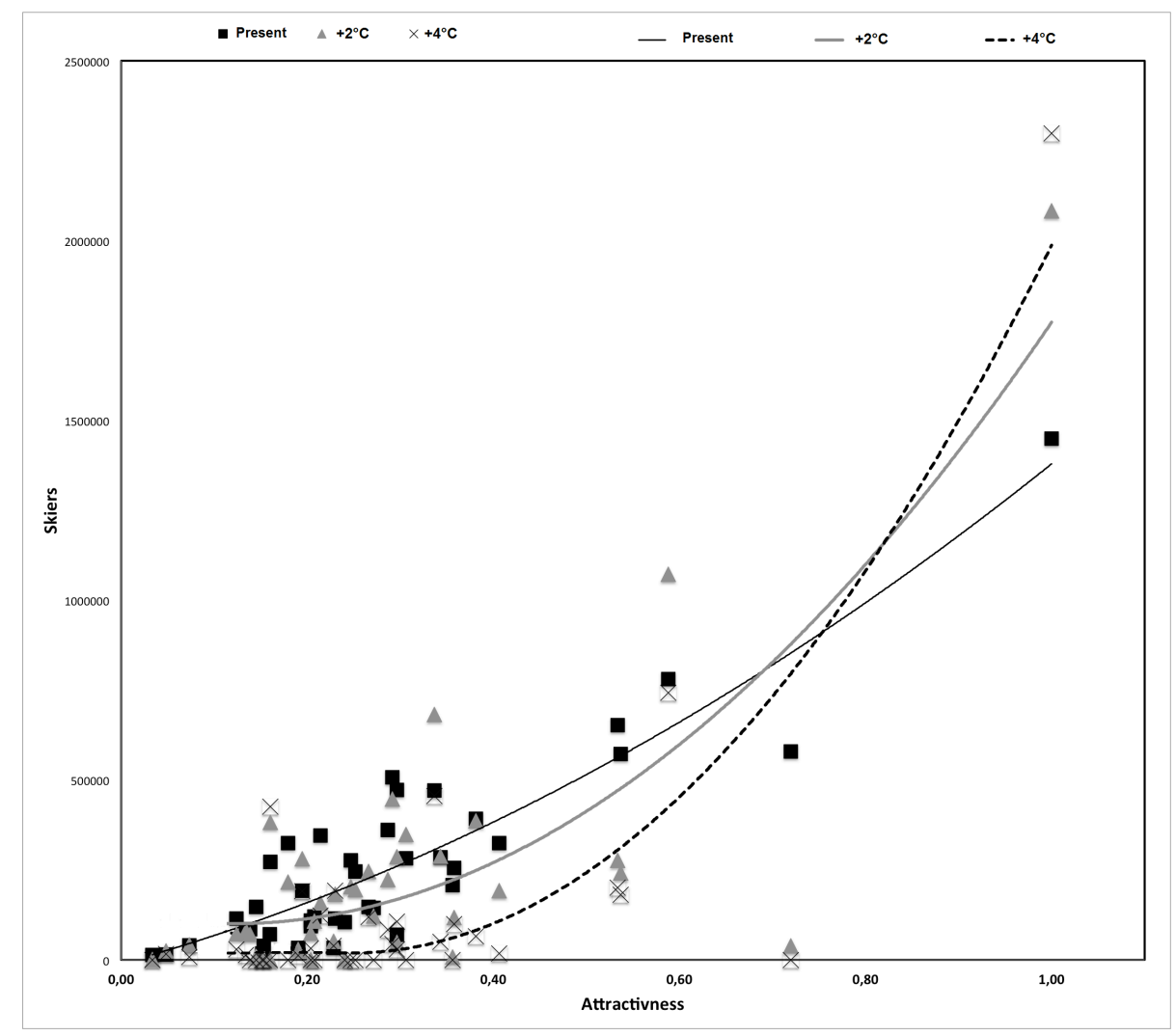

Figure 7. Attractiveness factor and changes in the total number of skiers for each ski resort in three different scenarios: a mean present winter season and assuming an increase of $+2^{\circ} \mathrm{C}$ and $+4^{\circ} \mathrm{C}$ of the winter mean temperature.

Figure 8, shows the attractiveness factor and the projected number of skiers in a present mean winter season and for two future scenarios: assuming an increase of $+2^{\circ} \mathrm{C}$ and 
$+4^{\circ} \mathrm{C}$ of the winter mean temperature and taking into account the capacity of snowmaking to increase season length.

In this case, three different groups of ski resorts can be identified based on the projected changes to skier attendance and resort attractiveness. The first group of resorts (Figure 10), characterized with a low attractiveness index value, show a slight reduction in future attendance for the $+2{ }^{\circ} \mathrm{C}$ scenario, and in some cases keeps the current attendance of skiers. With this group of resorts, despite taking snowmaking into account, significant decreases in skier visitation are projected for the $+4^{\circ} \mathrm{C}$ scenario. The second group of ski resorts, generally with a medium attractiveness index value, are able to increase their attendance in the $+2^{\circ} \mathrm{C}$ scenario but not in the $+4{ }^{\circ} \mathrm{C}$, where decreases are projected. Finally, the third group, typically with a higher attractiveness index, shows opposite behavior to the first group, being able to increase future attendance in both scenarios. This demonstrates an outcome of highly attractive ski resorts consolidating their dominant position at the expense of less attractive ski resorts, that may be struggling to adapt to changing snow conditions. Figure 9 shows an example of a ski resort classified in this third, 'resilient' group with a high attractiveness index and privileged geographical conditions. This resort has a high capacity to attract the skiers from the more vulnerable resorts located in the surrounding area. However, in snowmaking scenarios, the capacity to attract is reduced. In the $+2{ }^{\circ} \mathrm{C}$ scenario with snowmaking, the vulnerability of surrounding ski resorts is lower and therefore competitiveness is more evenly distributed among resorts. In the $+4{ }^{\circ} \mathrm{C}$ scenario with snowmaking this effect is not as evident due to the reductions of the potential snowmaking days. 


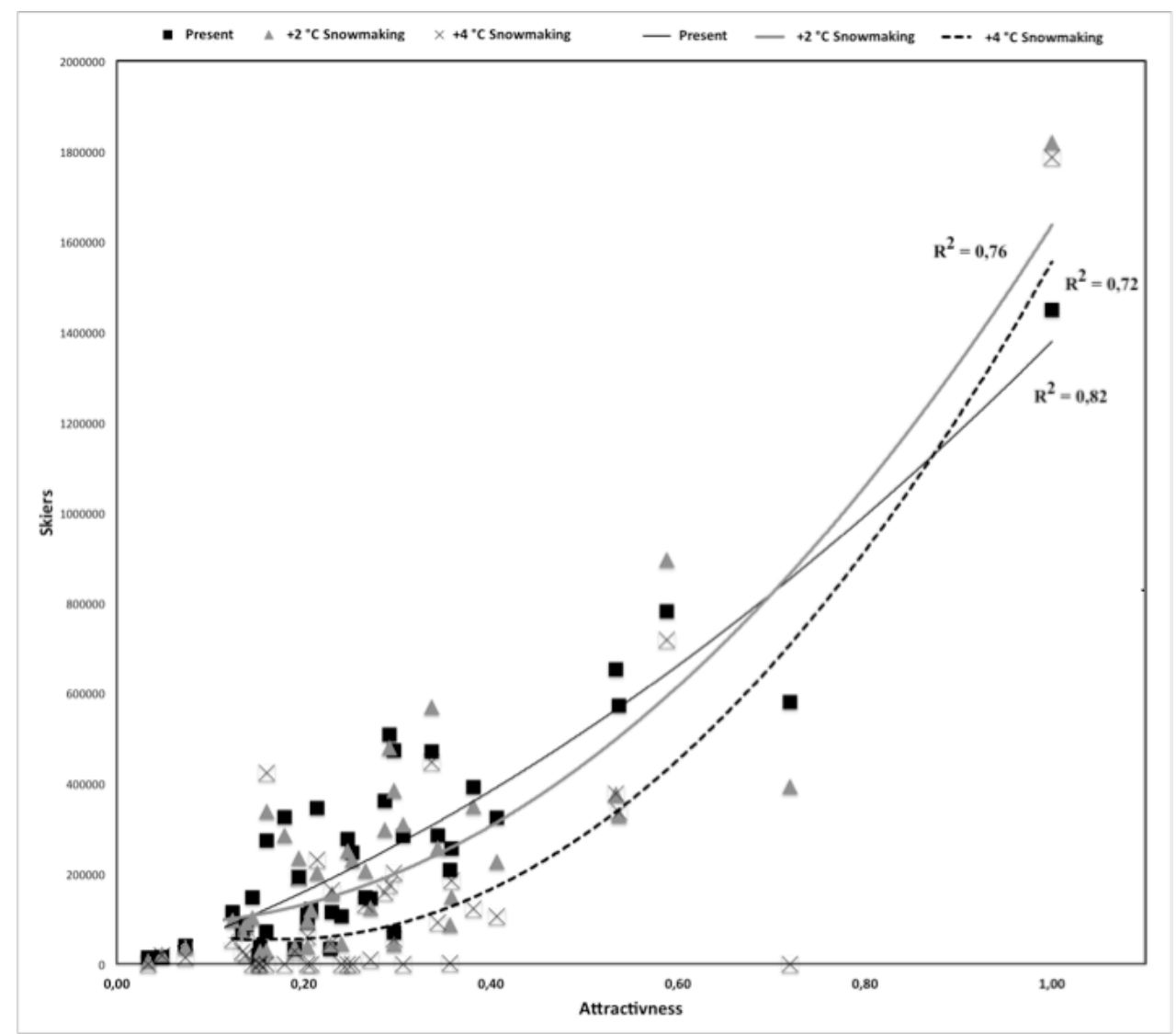

Figure 8. Attractiveness factor and changes in the total number of skiers for each ski resort in three different scenarios: a present winter season and assuming an increase of $+2^{\circ} \mathrm{C}$ and $+4^{\circ} \mathrm{C}$ of the winter mean temperature taking into account the contribution of snowmaking.

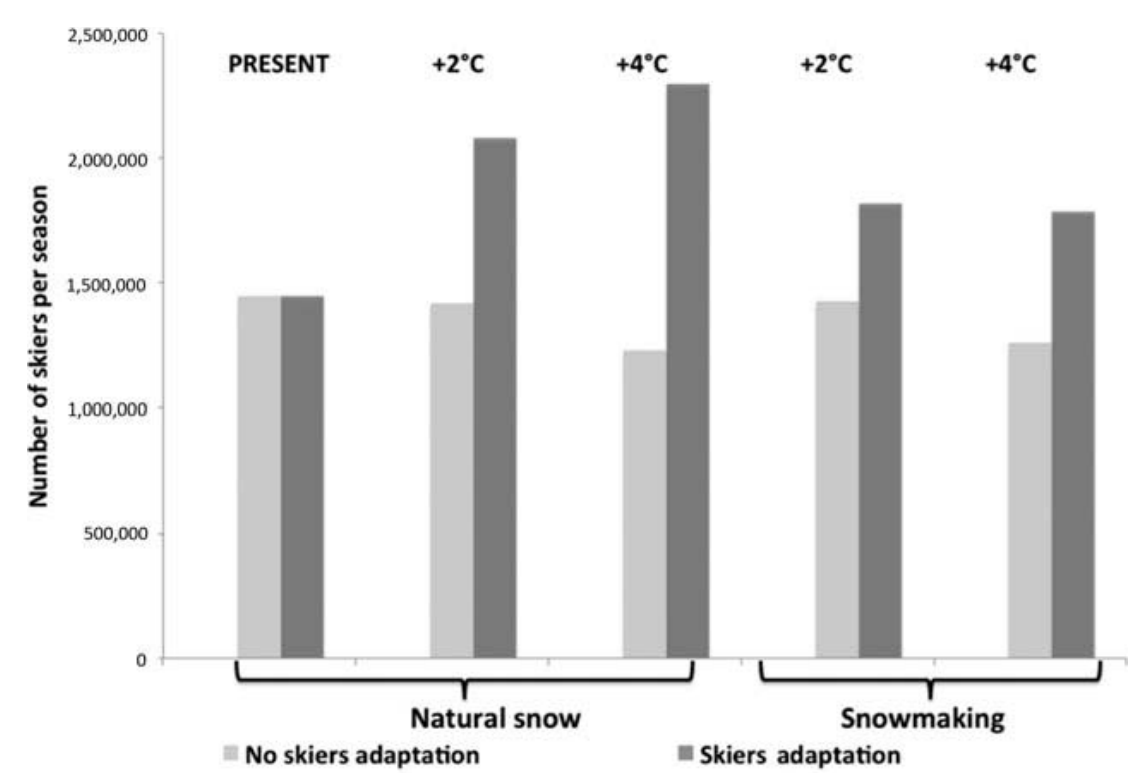

Figure 9. Yearly skier attendance in a resilient ski resort in different climate change scenarios with and without behavioral adaptation of skiers.

Regarding the relationship between the attractiveness factor and the total skier attendance in future climate change scenarios, a non-linear pattern is also observed when considering the effects of snowmaking (Figure 8). However, although increases in 
skier attendance are seen with every increase in attractiveness factor, snowmaking leads to a flattening of the curves with respect to the natural snow scenario, mainly in the $+2^{\circ} \mathrm{C}$ scenario. In the case of the $+4^{\circ} \mathrm{C}$ scenario, this effect is less significant since in a high climate change scenario, the effect of the snowmaking to enhance the season length is lower due to fewer potential snowmaking days available in the Pyrenean ski resorts. This change from an almost linear to an exponential relationship between the attractiveness and the total attendance of most of the analyzed ski resorts, results in an increase of the distance between the resilient and the vulnerable ski resort groups.

Figure 10 shows the clustering of the analyzed ski resorts in the 3 identified groups: the resilient ski resorts, the low vulnerability ski resort and high vulnerability ski resorts (only some ski resorts are shown in order to make the figure and the interpretation of the groups more clear).

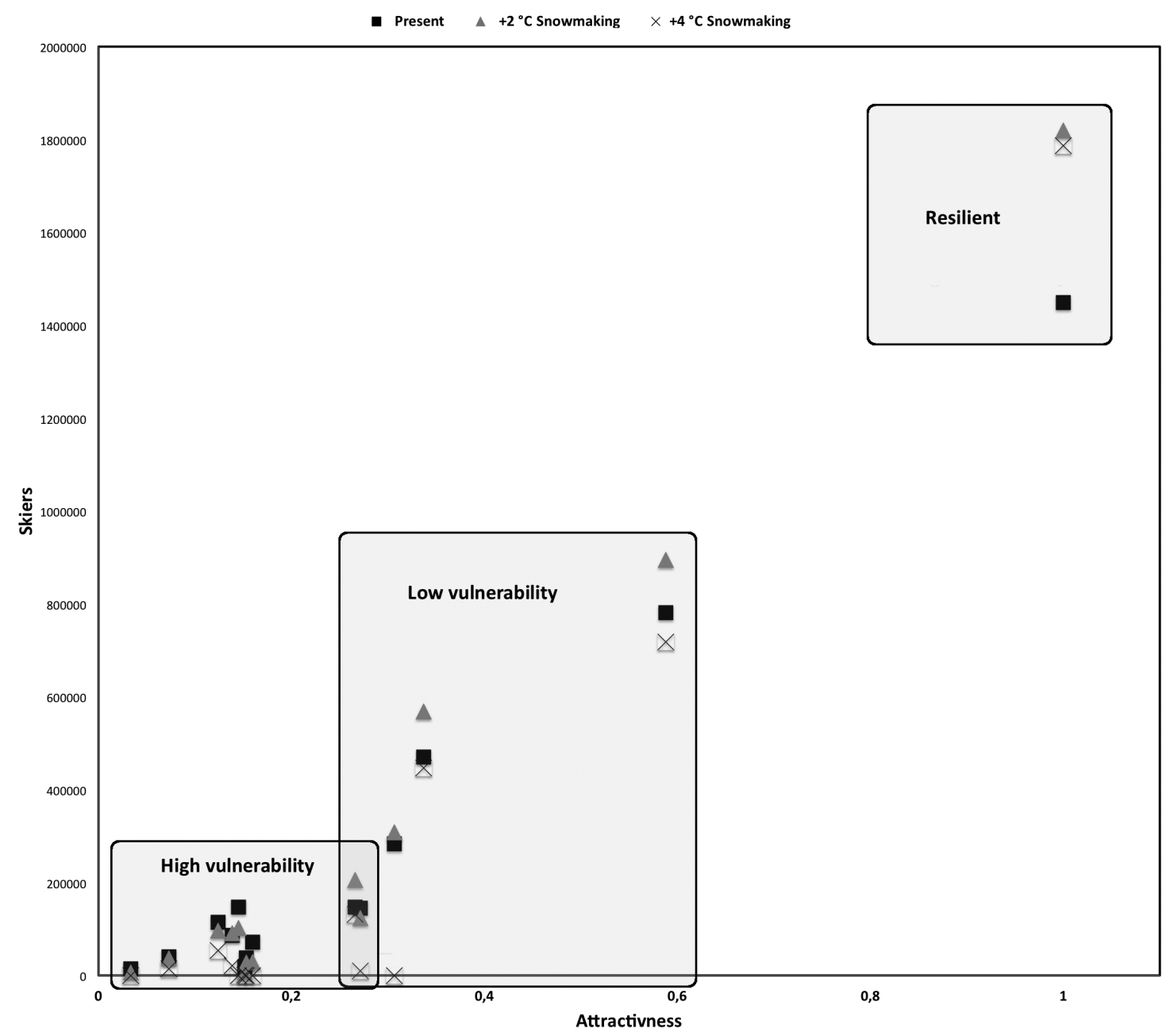

Figure 10. Clustering of the ski resorts in three groups: high vulnerable, low vulnerable, and resilient. 


\section{DISCUSSION}

The aim of the model presented here is to support an exploration of shifts in skier distribution amongst ski resorts at a regional macro-scale, due to changes in local snow conditions as a result of future climate change projections. To date, all studies analyzing the climate change impacts on ski resorts have assessed the potential reductions of skiers separately for each ski resort. This previous approach led in all the projected scenarios to a decrease in skier attendance for all the ski resorts (i.e., Scott et al. 2003, 2008, Dawson et al. 2009, Steiger 2010, Pons-Pons et al. 2012). In contrast with these studies, this approach presented here shows that the vulnerability of the ski resorts within the same geographical region can be affected by the response of skiers to poor snow conditions. The agent-based model permitted the inclusion of the individual behavioral response of skiers by means of an activity or spatial substitution when their typically frequented ski resort was closed due to poor snow conditions.

Results from this analysis are used to classify the ski resorts in three different groups. A first group consists of highly vulnerable ski resorts that will suffer an attendance reduction on its attendance of visitors in both mild and strong climate change scenarios. This group is usually characterized by conditions that make it difficult to ensure a snowreliable season, such as low elevation (Steiger, 2010; Pons-Pons et al, 2012), south oriented areas, with a predominant Mediterranean influence (Lopez-Moreno et al., 2009) and also a low touristic attractiveness compared to other nearby competitors. A second group consists of low vulnerability ski resorts that will suffer a reduction in attendance under a strong climate change scenario, but not in a mild one, where these resorts would keep their current level of skier attendance or even increase it. This group is usually characterized by ski resorts with medium capacity to assure enough snow and a medium attractiveness factor to capture skiers from other closed ski resorts. Lastly a third group consists of resilient ski resorts, with good conditions to assure future snow availability (high elevations, north oriented slopes, more Atlantic influence (LópezMoreno et al., 2009) and with a high attractiveness factor, which makes them able to offer longer ski seasons than their competitors and to attract skiers from closed ski resorts. Ski resorts classified in this group will increase their skier attendance in both low and high climate change scenarios. A new variable, the attractiveness of each ski resort, was included in the analysis of the climate change impacts on the winter tourism. By means of a gravity model, this variable, characterized by structural and supply features of each ski resort, allows, together with distances between ski resorts, to project the potential redistribution of skiers. This attractiveness factor is found to affect the vulnerability of each ski resort. Ski resorts able to offer longer ski seasons compared to other ski resorts, plus having a high attractiveness factor are considered to be the most resilient to climate change impacts.

The second parameter of the gravity model, the travel distance between ski resorts was found not to have a huge impact in the particular case study of the Pyrenees. In this case, the shifting options for skiers, and consequently the sensitivity to alpha in 
$D i j^{\alpha}$, are highly reduced due to the resulting configuration of vulnerable and resilient ski resorts to climate change. This is because the redistribution is performed mainly and only among the limited resilient resorts, which also stand as the more attractive ones and with already a high attendance on comparison with the attracted skiers. However, when testing the model sensitivity to $\alpha$ under less severe conditions (i.e., homogenous attractiveness and variability in the projected season length reduction), this variable becomes a significant parameter to take into account in the model. Therefore, it has to be considered to apply this model to other regions and correctly calibrate the model.

Due to the low frequency in the occurrence of years with analogous conditions to future climate scenarios and the scarcity of detailed historical data of skier attendance at each ski resort, the validation of the model results is a central challenge to the modeling process. Despite this constraint, the error of the model is acceptable for a first stage of development. There are several key sources of error that can affect the model outcomes. First, there is inherent error of the snow cover regional model used and its resolution to capture enough local variability. Second, because of high competition between ski resorts, there is limited availability of the skier attendance data, and its reliability should be questioned. This unreliability of resort-sourced data is an issue that could compromise the comparison of projected skiers attendance with the analogous year. Finally, since there is no Pyrenean survey so far capturing the adaptive behavior of skiers when confronted with poor snow conditions, results of surveys from different studies in the Alps (Behringer et al., 2000) and USA (Dawson, 2011) were used. In addition to the share of temporal substitution, not taken into account in this study, this issue could also introduce a significant error to the model because the dynamics and the response of the Pyrenean tourists may be different.

Future areas of refinement of this work will focus on reducing the error of the model and improve the accuracy of the projected results. To achieve this we are working to create and use snow cover models with higher spatial resolution, able to better capture the regional variability at a local scale in the future snow depth of each ski resort. Additionally, a survey will be designed and carried out to capture the behavioral response of visitors when facing poor snow conditions for the Pyrenees region. Moreover, the segregation of different adaptative behaviors based on different skier profiles and the influence of the future scarcity of resources like fuel or water, or the effect of crowdedness will be considered in order to analyze potential sensible variables that could constraint the ski industry. One of the main limitations of the current model version is the use of the distance. The suitable distance to consider when modeling skiers redistribution should be the distance between the skier home origin and the selected ski resort. Our initial purpose was to build the gravity model or the radiation model (Simini, et al., 2012) using this information. Even though the distance between resorts does not exactly represent the reality of the dynamic with the available data it was the only solution to include in this first version the distance effect on the decision process. To overcome this limitation, a future survey will gather information about the 
origin and travel behavior of skiers to consider skier home origin and not the frequented ski resort. Finally, the gravity approach, lacking a powerful theoretical guidance, relies on adjustable parameters to fit empirical data that vary from region to region. In this line, non-parametric approaches like the radiation model (Simini et al., 2012.) could be tested in a future in order to improve the projected redistribution of skiers among the remaining ski resorts.

\section{CONLUSIONS}

A georeferenced ABM methodology coupled with a gravity model was used to simulate climate change impacts on winter tourism, and specifically used to analyze the interaction between physical changes (i.e., snowpack and resulting season length) and socioeconomic implications (i.e., potential shifts in number of skiers). Unlike many of the models used to date to analyze climate change impacts on ski industry, this methodology permits the inclusion of behavioral response of skiers. The study assessed the effects of spatial and activity substitution on the shifts in the future redistribution of skiers among the ski resorts at a regional scale. This study proposes a new approach to better understand and explore how the potential behavioral responses identified in previous studies could affect the final outcome of climate change impacts assessment. In addition to the identified behaviors and responses of tourists, this framework could be also useful in a future to explore the influence of tourist behavior and responses to other factors that could affect their dynamics such as petrol price or crowding avoidance in destinations once the appropriate information have been generated and available.

Thus, this approach fosters the understanding of the climate change impacts on winter tourism in any region of the world, by means of a better characterization of the variability of the ski resorts vulnerability in a close geographical area including the effect of the behavioral adaptation of tourists. The high variability detected on the level of impacts at short distances leads not to a significant regional pattern about the vulnerability. In this line, we found that in the same region can be two resorts located at a really short distance with significant different level of vulnerability. However and congruent with previous cited literature, low elevation areas, with a predominance of south oriented slopes present a higher vulnerability. In the particular case of the Pyrenees, those resorts with a higher Mediterranean influence and those with little diversity of complementary activities to ski were identified as the more vulnerable to future climate change. Otherwise, those with a higher Atlantic influence, located at higher elevations, more north oriented and with a more diversified complementary to ski activities were identified as the more resilient ones. In the case of the Pyrenean region, our results show that, even more than a significant reduction in the ski market, the main effect of climate change will be a redistribution and consolidation of the current market, reallocating the visitors of more vulnerable ski resorts to the most resilient ones, able to offer longer ski seasons plus having a higher attractiveness factor than their competitors. Furthermore, the clustering of the ski resorts on the basis of their vulnerability is of a great value for managers and policymakers in order to identify 
targets to focus the efforts and design the suitable measures for adaptation strategies for each ski resort.

\section{Acknowledgements}

The authors are thankful to Dr. Ignacio López-Moreno for share and support in climate projections data and to the Working Community of the Pyrenees (CTP) research projects CTTP $1 / 10$ and CTTP1/12, financially supported by the Government of Andorra. First author acknowledges also a predoctoral grant from the Andorran Government [BTC2010/2013-0006-AND].

\section{BIBLIOGRAPHY}

Abegg, B. 1996. Klimaänderung und Tourismus. Klimafolgenforschung am Beispiel des Wintertourismus in den Schweizer Alpen. vdf Hochschuleverlag, Zurich.

Abegg B, Agrawala S, Crick F, de Montfalcon, A. 2007. Climate change impacts and adaptation in winter tourism. In: Climate change in the European Alps: adapting winter tourism and natural hazards management. Paris: Organization for Economic Co-operation and Development.

Alexandru, A., de Elía, R., Laprise, R. 2007. Internal variability in regional climate downscaling at the seasonal time scale. Mon Weather Rev 135(9), 3221-3238

Andorra Turisme. 2012. National Tourism Surveys. Andorra Government.

ATUDEM. 2013 Dossier de premsa 2012-2013. Associación Tursítica de Estaciones De Esquí y Montaña.

Axtell, R. L., Epstein, J. M., Dean, J. S., Gumerman, G. J., Swedlund, A. C., Harburger, J., Chakravarty, S., Hammond, R., Parker, J., Parker, M. 2002. Population Growth and Collapse in a Multi-Agent Model of the Kayenta Anasazi in Long House Valley. Proceedings of the National Academy of Sciences 99(3), 7275-7279.

Baggio, R. 2008. Symptoms of complexity in a tourism system. Tourism Analysis $13,1-20$

Balbi, S., Giupponi, C., Perez, P., Alberti, M. 2013. A spatial agent-based model for assessing strategies of adaptation to climate and tourism demand changes in an alpine tourism destination. Environmental Modelling \& Software, 45,29 - 51

Bark, R., Colby, B., Dominguez, F. 2010. Snow days? Snowmaking adaptation and the future of low latitude, high elevation skiing in Arizona, USA. Climatic Change 102, 467-491. 
Becken, S. 2005. Harmonising climate change adaptation and mitigation: The case of tourist resorts in Fiji. Global Environmental Change, 15 (4), 381-393

Behringer, J., Bürki, R., Fuhrer, J. 2000. Participatory integrated assessment of adaptation to climate change in alpine tourism and mountain agriculture. Integrated Assessment 1(3), 331338

Bithell, M., Brasington, J., 2009. Coupling agent-based models of subsistence farming with individual-based forest models and dynamic models of water distribution. Environmental Modelling \& Software 24 (2), 173-190.

Botti, L., Goncalves, O., Peypoch, N. 2013. Benchmarking Pyrenean ski resorts. Journal of Alpine Research, 100-4.

Crooks, A. T., 2010. Constructing and Implementing an Agent-Based Model of Residential Segregation through Vector GIS. International Journal of Geographical Information Science, 24(5), 661-675.

Dawson, J., 2009. Climate Change Vulnerability of the US Northeast Ski Sector: A multimethods systems-based approach. Thesis (PhD) Department of Geography and Environmental Management, University of Waterloo.

Dawson, J., Scott, D., McBoyle, G. 2009. Climate change analogue analysis of ski tourism in the northeastern USA. Clim Res 39,1-9

Dawson, J., Havitz, M., Scott, D., 2011. Behavioral Adaptation of Alpine Skiers to Climate Change: Examining Activity Involvement and Place Loyalty. Journal of Travel \& Tourism Marketing, 28 (4), 388-404.

Dawson, J., Scott, D. (2012) Managing for Climate Change in the Alpine Ski Sector. Tourism Management. 35, 244-254.

Dawson, J., Scott, D., Havitz, M. 2013. Skier demand and behavioural adaptation to climate change in the US Northeast. Leisure/ Loisir, 37(2), 127-143.

DSF. 2012. Recueil d'indicateurs et analyses 2012. Domaines Skiables de France

Ford, J.D., Keskitalo, E.C.h:, Smith, T., Pearce, T., Berrang-Ford, L., Duerden., F. and Smit, B. 2010. Case study and analogue methodologies in climate change vulnerability research. WIRE's Climate Change, 1(3):374-392.

Fukushima, T., Kureha, M., Ozaki, N., Fujimori, Y., Harasawa, H., 2002. Influences of air temperature change on leisure industries: case study on ski activities. Mitigation and Strategies for Climate Change 7:173-189.

Gimblett, R., Skov-Petersen, H., 2008. Monitoring, Simulation, and Management of Visitor Landscapes. University of Arizona, Tuscon. 
Grimm, V., U. Berger, F. Bastiansen, S. Eliassen, V. Ginot, J. Giske, J. Goss-Custard, Grand, T., Heinz, S. K., and Huse, G. 2006. A standard protocol for describing individual-based and agent-based models. Ecological Modelling 198(1-2), 115-126.

Grimm, V., Berger, U., DeAngelis, D.L., Polhill, J.G., Giske, J., Railsback, S.F. 2010. The ODD protocol for describing individual-based and agent-based models: A first update. Ecological Modelling, 221, 2760-2768.

Gössling, S., D. Scott, C., Michael Hall, J.-P. Ceron and Dubois, G. 2012. Consumer behaviour and demand response of tourists to climate change. Annals of Tourism Research, 39(1), 36-58.

Hamilton, L, Brown, C., \& Keim, B. D. 2007. Ski areas, weather and climate: Time series models for New England case studies. International Journal of Climatology, 27, 2213-2124.

Hennessy, K., Whetton, P., Smith, I., Bathols, J., Hutchinson, M., Sharples, J. 2003. The impact of climate change on snow conditions in mainland Australia. CSIRO Atmospheric Research. Aspendale, Australia

Isard, W. 1960. Methods of regional analysis: an introduction to regional science. The MIT Press, Cambridge.

Hoffmann, V H; Sprengel, D C; Ziegler, A; Kolb, M; Abegg, B. 2009. Determinants of corporate adaptation to climate change in winter tourism: an econometric analysis. Global Environmental Change, 19(2), 256 -264.

IPCC. 2007. Climate change 2007: Impacts, adaptation and vulnerability. Contribution of Working Group II to the Fourth Assessment Report of the Intergovernmental Panel on Climate Change. Cambridge, United Kingdom \& New York, NY, USA: Cambridge University Press.

Itami, R., Raulings, R., MacLaren, G., Hirst, K., Gimblett, R., Zanon, D., Chladek, P. 2002. RBSim 2: Simulating the complex interactions between human movement and the outdoor recreation environment. In Monitoring and Management of Visitor Flows in Recreational and Protected Areas. Conference Proceedings, A. Arnberger, C. Brandenburg, A. Muhar, Institute for Landscape Architecture and Landscape Management University of Agricultural Sciences, Vienna, Austria, 30 Jan - 02 Feb 2002 pp 191-198.

Janssen, M. A. 2009. Understanding artificial anasazi. Journal of Artificial Societies and Social Simulation, 12.

Johnson, P. A., Sieber, R. E. 2009. Agent-based modelling: A dynamic scenario planning approach to tourism PSS. In S. Geertman \& J. Stillwell (Eds.), Planning Support Systems: Best Practices and New Methods (pp. 211-226). Berlin: Springer.

Johnson, P. A., Sieber, R. E. 2010. An Individual-Based Approach to Modeling Tourism Dynamics. Tourism Analysis, 15(5),517-530.

Johnson, P. A., Sieber, R. E. 2011. An Agent-Based Approach to Providing Tourism Planning 
Support. Environment and Planning B - Planning and Design, 38(3), 486-504.

Jung, W. S., Wang, F., Stanley, H. E. 2008. Gravity model in the Korean highway. EPL 81, 48005.

Kaluza, P., Kölzsch, A., Gastner, M. T., Blasius, B. 2010. The complex network of global cargo ship movements. J. R. Soc. Interf. 7, 1093-1103.

Karemera, D., Oguledo, V.I., Davis, B. 2000. A gravity model analysis of international migration to NorthAmerica. Appl Econ 32(13), 1745-1755.

Kohler, T. A., Gumerman, G. J. 1999. Dynamics in human and primate societies: Agent-based modeling of social and spatial processes. New York: Oxford University Press.

Krings, G., Calabrese, F., Ratti, C., Blondel, V. D. 2009. Urban gravity: a model for intercity telecommunication flows. J. Stat. Mech, L07003.

Lamothe and Périard Consultants.1988. Implications of climate change for downhill skiing in Québec. Climate Change Digest 88-03. Environment Canada, Ottawa

Ligmann-Zielinska, A., Jankowski, P. 2007. Agent-based models as laboratories for spatially explicit planning policies. Environment and Planning B, 34,316-335

March, H., Saurí, D., Llurdés, J.C. 2014. Perceptions on the effects of climate change in winter and summer tourists areas: The Pyrenees and the Catalan and Balearic coasts, Spain. Regional Environmental Change, 14(3), 1189-1201.

López-Moreno, J.L, Goyette, S., Beniston, M. 2009. Impact of climate change on snowpack in the Pyrenees: horizontal spatial variability and vertical gradients. $J$ Hydrol (Amst) 374, 384-396

Manson, S. M., Evans, T. 2007. Agent-based modeling of deforestation in southern yucatán, mexico, and reforestation in the midwest united states. Proceedings of the National Academy of Sciences, 104(52), 20678-20683.

Moen, J., Fredman, P. 2007. Effects of climate change on alpine skiing in Sweden. Journal of sustainable tourism 15: 418-437.

Monge, G. 1781. Mémoire sur la Théorie des Déblais et de Remblais. Histoire de l'Académie Royale des Sciences de Paris, avec les Mémoires de Mathématique et de Physique pour la même année 666-704 (De l'Imprimerie Royale, 1781).

Parker, D.C., Manson, S. M., Janssen, M. A., Hoffmann, M. J., Deadman, P., 2003. Multi-agent systems for the simulation of land-use and land-cover change: a review. Annals of the Association of American Geographers 93(2), 314-337.

Perez, L., Dragicevic, S. 2010. Environmental Modelling \& Software 25 (2), 223-236

Pons-Pons, M., Johnson, P.A., Rosas-Casals, M., Sureda, B., Jover, È. 2012. Modeling climate 
change effects on winter ski tourism in Andorra. Clim Res 54, 197-207

Pöyhönen, P. 1963. A tentative model for the volume of trade between countries. Weltwirtschaftliches Arch. 90, 93-100.

Pütz, M., Gallati, D., Kytzia, S., Elsasser, H. and others 2011. Winter tourism, climate change, and snowmaking in the Swiss Alps: tourists' attitudes and regional economic impacts. Mt Res Dev 28, 292-298

Scott, D., McBoyle, G., Mills, B. 2003. Climate change and the skiing industry in southern Ontario (Canada): exploring the importance of snowmaking as a technical adaptation. Clim Res $23,171-181$

Scott, D, McBoyle, G., Mills, B., \& Minogue, A. 2006. Climate change and sustainability of ski-based tourism in eastern North America: A reassessment. Journal of Sustainable Tourism, 14(4), 376-398

Scott, D., Dawson, J., Jones, B. 2008. Climate change vulnerability of the US northeast winter recreation- tourism sector. Mitigation and Adaptation Strategies for Global Change 13 (5), 577-596.

Scott, D., Gössling, S., Hall, C.M. 2012, Climate Change and Tourism: Impacts, Adaptation and Mitigation. London: Routledge. 423 pgs.

Simini, F., González, M., Maritan, A., Barabási, A.-L., 2012. A universal model for mobility and migration patterns. Nature, 484, 96-100.

Ski Andorra. www.skiandorra.ad. Last access November 21st 2013.

Smajgl, A., Heckbert, S., Ward, J., Straton, A., 2009. Simulating impacts of water trading in an institutional perspective. Environmental Modelling \& Software 24(2), 191-201.

Steiger, R. 2010. The impact of climate change on ski season length and snowmaking requirements in Tyrol, Austria. Clim Res 43, 251-262

Steiger, R. (2011). The impact of snow scarcity on tourism. An analysis if the record warm seson 2006/2007 in Tyrol (Austria). Tourism Review 66(3):4-13

Steiger R, Abegg B. 2013. The sensitivity of Austrian Ski areas to climate change. Tourism, planning and development, 480-493.

Thiemann, C., Theis, F., Grady, D., Brune, R. \& Brockmann, D. 2010. The structure of borders in a small world. PLOS ONE 5, e15422.

Uhlmann, B., Goyette, S., Beniston, M. 2009. Sensitivity analysis of snow patterns in Swiss ski resorts to shifts in temperature precipitation and humidity under condition of climate change. International Journal of Climatology 29, 1048-1055 
Unbehaun, W., Probstl, U., \& Haider, W. 2008. Trends in winter sports tourism: Challenges for the future. Tourism Review, 63(1), 36-47.

Viaggi, D., Bartolini, F., Raggi, M. 2009. Combining linear programming and principal-agent models: an example from environmental regulation in agriculture. Environmental Modelling \& Software 24 (6), 703-710.

Zipf, G. K. 1946. The P1P2/D hypothesis: on the intercity movement of persons. Am. Sociol. Rev. 11, 677-686. 